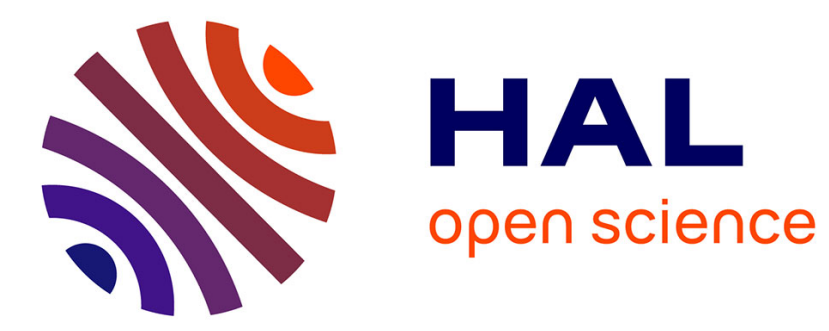

\title{
De l'allégeance à la rue de Rivoli au pèlerinage de Wall Street. Financiarisation et professionnalisation des directeurs financiers dans l'énergie et la défense
}

\author{
Hadrien Coutant, Scott Viallet-Thevenin
}

\section{- To cite this version: \\ Hadrien Coutant, Scott Viallet-Thevenin. De l'allégeance à la rue de Rivoli au pèlerinage de Wall Street. Financiarisation et professionnalisation des directeurs financiers dans l'énergie et la défense. Entreprises et Histoire, 2019, n 95 (2), pp.40-54. 10.3917/eh.095.0040 hal-02423918}

\section{HAL Id: hal-02423918 \\ https://hal.science/hal-02423918}

Submitted on 26 Dec 2019

HAL is a multi-disciplinary open access archive for the deposit and dissemination of scientific research documents, whether they are published or not. The documents may come from teaching and research institutions in France or abroad, or from public or private research centers.
L'archive ouverte pluridisciplinaire HAL, est destinée au dépôt et à la diffusion de documents scientifiques de niveau recherche, publiés ou non, émanant des établissements d'enseignement et de recherche français ou étrangers, des laboratoires publics ou privés. 


\section{Titre}

De l'allégeance à la rue de Rivoli au pèlerinage de Wall Street.

Financiarisation et professionnalisation des directeurs financiers dans l'énergie et la défense.

\section{Mots clés}

Financiarisation, entreprise publique, État, $\mathrm{XX}^{\text {ème }}$ siècle, dirigeants d'entreprise

\section{Auteurs}

Hadrien Coutant (hadrien.coutant@ sciencespo.fr)

Scott Viallet-Thévenin (scott.vialletthevenin@ sciencespo.fr)

\section{Biographies des auteurs}

Hadrien Coutant est sociologue, post-doctorant au Centre de Sociologie des Organisations (CNRS/Sciences Po). Ses recherches portent sur les transformations du capitalisme contemporain à travers l'étude des grandes firmes, en articulant sociologie des marchés, du travail et des organisations.

Scott Viallet-Thévenin est sociologue, post-doctorant au labex SMS (Université de Toulouse Jean Jaurès), chercheur associé au CSO (Sciences Po). Ses recherches portent sur les relations entre élites économiques et administratives, dans le cas du secteur de l'énergie contemporain et de l'Empire colonial français.

\section{Chapeau}

À partir d'une étude des trajectoires et positions des directeurs financiers des entreprises historiquement publiques de l'énergie et de l'aéronautique, cet article montre comment la financiarisation provoque leur renforcement et leur professionnalisation. Avec des rythmes différents, ces entreprises participent à un même processus qui accompagne leur exposition croissante aux marchés financiers et aux transformations de l'État actionnaire.

\section{Résumé}

L'histoire des directions financières des entreprises de l'énergie et de la défense donne à voir un renforcement et une professionnalisation croissante des directeurs financiers. Spécialisation dans la finance, puis dans le poste de directeur financier, internationalisation et carrières combinant industrie financière et finance d'entreprise ont profondément transformé le profil des directeurs financiers. Cette transformation des individus et de leur position dans l'organisation a permis aux entreprises partiellement ou totalement privatisées de construire leur légitimité financière. Les entreprises ont pu ainsi s'assurer la confiance des investisseurs institutionnels dont la part croissait dans le capital. Elles ont également pu répondre à la financiarisation croissante de l'État actionnaire lui-même sous l'impulsion de l'Agence des Participation de l'État créée par le Ministère des Finances en 2004. 


\section{De l'allégeance à la rue de Rivoli ${ }^{1}$ au pèlerinage de Wall Street. Financiarisation et professionnalisation des directeurs financiers dans l'énergie et la défense.}

\section{INTRODUCTION}

La financiarisation des firmes depuis les années $1980^{2}$ est un processus multidimensionnel de transformation de l'économie. Outre des dimensions structurelles (le développement des investisseurs institutionnels), institutionnelles (la libéralisation des marchés financiers) et idéologiques (la théorie de l'agence ${ }^{3}$ ), elle comprend des dynamiques internes aux entreprises. Les directions financières, bien qu'anciennes au sein de nombreuses grandes entreprises françaises, ont connu au cours des quarante dernières années des transformations spectaculaires que nous proposons d'approcher à travers celles de leurs dirigeants, les directeurs financiers. La littérature a montré un renforcement de la position des directeurs financiers et des contrôleurs de gestion dans la direction des firmes ${ }^{4}$, ainsi que l'accroissement du nombre d'individus issus de la finance aux postes de direction. Cette dynamique est utilisée par Neil Fligstein ${ }^{5}$ comme un indicateur de financiarisation. Les travaux existants ont généralement une dimension macrosociologique, qui permet de montrer le caractère structurel de ces transformations, mais qui suppose de poser des hypothèses fortes sur l'effet de la trajectoire des directeurs financiers sur la stratégie des firmes. Par ailleurs le cas français reste peu étudié, ce qui a amené à une surestimation du caractère importé de la financiarisation, récemment remise en cause ${ }^{6}$. La proximité d'une part importante des grandes entreprises avec l'État, notamment par la propriété publique du capital, a pu être perçue comme

${ }^{1}$ Le Ministère de l'Économie et des Finances a déménagé de la rue de Rivoli à Bercy entre 1987 et 1989.

${ }^{2}$ G. Davis et T. Thompson, «A Social Movement Perspective on Corporate Control», Administrative Science Quarterly, 39, $\mathrm{n}^{\circ} 1,1994$, p. 141-173 ; N. Fligstein et T. Shin, «Shareholder Value and the Transformation of the U.S. Economy, 1984-2001», Sociological Forum, 22, nº 4, décembre, 2007, p. 399-424; J. Froud, C. Haslam, S. Johal et K. Williams, "Shareholder value and Financialization: consultancy promises, management moves », Economy and Society, 29, $\mathrm{n}^{\circ} 1$, 2000, p. 80-110 ; G. Krippner, "The Financialization of the American Economy », Socio-Economic Review, 3, n², 2005, p. 173-208; E. Zuckerman, «Focusing the Corporate Product: Securities Analysts and De-Diversification », Administrative Science Quarterly, 45, n³, 2000, p. 591-619 ; N. van der Zwan, « Making Sense of Financialization », Socio-Economic Review, 12, nº1, 2014, p. 99-129.

${ }^{3}$ J. Jung et F. Dobbin, « Agency Theory as Prophecy: How Boards, Analysts, and Fund Managers Perform Their Roles », Seattle University Law Review, 39, 2016, p. 291-320.

${ }^{4}$ J. Kädtler et H. Sperling, «The Power of Financial Markets and the Resilience of Operations: Argument and Evidence from the German Car Industry », Competition \& Change, 6, $\mathrm{n}^{\circ} 1,2002$, p. 81-94 ; J. Morales et A. Pezet, "Les contrôleurs de gestion, "médiateurs» de la financiarisation. Étude ethnographique d'une entreprise de l'industrie aéronautique », Comptabilité - Contrôle - Audit, 16, nº1, 2010, p. 101-132 ; D. Zorn, "Here a Chief, There a Chief: The Rise of the CFO in the American Firm », American Sociological Review, 69, no3, 2004, p. 345-364.

${ }^{5}$ N. Fligstein, «The Intraorganizational Power Struggle: Rise of Finance Personnel to Top Leadership in Large Corporations, 1919-1979», American Sociological Review, 52, $\mathrm{n}^{\circ} 1$, 1987, p. 44-58; N. Fligstein, The Transformation of Corporate Control, Cambridge, Mass., Harvard University Press, 1990.

6 F. Foureault, "L'organisation de la financiarisation. Structure et développement du champ des fonds d'investissement en France », Revue française de sociologie, 59, n 1, 2018, p. 37-69 ; P. François et C. Lemercier, « Une financiarisation à la française (1979-2009), Mutations des grandes entreprises et conversion des élites », Revue française de sociologie, 57, n², 2016, p. 269-320. 
une protection contre le mouvement de financiarisation. Étudier la professionnalisation de leurs directeurs financiers permet de s'interroger sur l'étendue de la financiarisation de ces entreprises et de discuter le caractère supposé protecteur du capital public.

Par professionnalisation, nous entendons l'émergence progressive d'un groupe professionnel, aux carrières présentant des similitudes, autour d'une juridiction commune ${ }^{7}$, c'est-à-dire un ensemble de savoirs et connaissances qui permettent à ces membres de revendiquer la légitimité de leurs pratiques par rapport à d'autres individus constitués ou non en professions. En suivant Andrew $\mathrm{Abbott}^{8}$, on fait l'hypothèse que ces groupes se constituent à travers l'édification de frontières locales, vis-à-vis de groupes proches. Ainsi des frontières émergent localement avec d'autres groupes professionnels qui peuvent servir de base à la formation d'un nouveau groupe professionnel. Dans le cas des directeurs financiers, cette spécialisation et autonomisation se fait vis-à-vis d'autres dirigeants de la firme, liés à l'administration ou issus d'autres fonctions de l'entreprise. Après une période où les directeurs financiers bénéficiaient de leur lien à l'État ou de longues carrières internes, ils se sont spécialisés dans des fonctions financières, avant de s'autonomiser des entreprises elles-mêmes en passant au cours de leur carrière au sein de nombreuses entreprises.

Afin d'étudier les transformations des directions financières de grandes entreprises contemporaines, nous proposons une analyse diachronique qualitative de l'évolution des carrières et positions occupées par les directeurs financiers dans deux secteurs économiques marqués par une grande proximité avec l'État: la défense et l'énergie'. Le capital des grandes entreprises de ces secteurs était protégé par un actionnariat stable et public jusqu'au début des années 2000, période durant laquelle elles ont été confrontées à des processus de transformation de leur actionnariat (privatisations), de leurs stratégies (internationalisation), et de leur morphologie (fusions et acquisitions). Nous avons construit une base de données renseignant les parcours des directeurs financiers successifs des entreprises sur la période 1985-2015. Nous nous appuyons également sur des entretiens réalisés auprès de dirigeants des entreprises et de hauts fonctionnaires en 2012 et 2013, ainsi que sur une abondante littérature grise (rapports financiers et documents de références des entreprises, rapports de l'Agence des Participations de l'État, articles de presse économique). Le choix de se concentrer sur deux secteurs et un nombre réduit d'entreprises nous permet de préciser les carrières des directeurs financiers et leur position, ainsi que de proposer un modèle processuel de la montée en puissance des directions financières dans les firmes. Ce modèle construit de manière inductive montre les différents aspects de cette montée en puissance et les importantes différences de rythmes entre firmes et entre secteurs.

Le secteur de l'énergie français ${ }^{10}$ est marqué depuis 1946 par l'intervention de l'État et les arrangements néo-corporatistes qui lient les entreprises aux directions et aux corps chargés de leur tutelle.

\footnotetext{
${ }^{7}$ A. Abbott, The System of Professions - An Essay on the Division of Expert Labor, Chicago, University of Chicago Press, 1988.

${ }^{8}$ A. Abbott, «Things of Boundaries », Social Research, 62, nº4, 1995, p. 857-882.

${ }^{9} \mathrm{H}$. Coutant, Un capitalisme d'ingénieurs. Construire un groupe aéronautique après une fusion, Paris, Sciences Po, Thèse en Sociologie, 2016; S. Viallet-Thévenin, Structurer un secteur industriel. Le rôle de l'État dans la recomposition du secteur de l'énergie en France, de 1986 à 2016, Paris, Sciences Po, Thèse en Sociologie, 2016.

${ }^{10}$ Les entreprises considérées sont, pour l'énergie: Framatome (1981-2001), la Cogéma (1976-2001), puis Areva (2001-2015) ; EDF (1946-) ; Gaz de France (1946-2004), devenu GDF-Suez (2004-2015) puis Engie (2015) ; la CFP
} 
La quasi-totalité des grandes entreprises du secteur de l'énergie des années 1980 a été créée par l'administration ou ses agences (Institut français du pétrole, Commissariat à l'énergie atomique). Leur création résulte d'une politique volontariste de l'État visant à pallier le manque de ressources énergétiques sur le territoire français et à assurer les approvisionnements en matières premières nécessaires à la production d'énergie ${ }^{11}$. Le secteur de l'énergie a été choisi en raison des transformations qui le caractérisent depuis le début des années 1980 : privatisations, libéralisations des marchés de l'électricité et du gaz, larges mouvements de fusions et acquisitions consécutifs à des stratégies d'internationalisation. L'aéronautique et la défense ${ }^{12}$ forment un secteur moins cohérent que l'énergie avec, depuis 1946, un ensemble d'entreprises publiques, d'arsenaux et d'entreprises privées très proches de l'État ${ }^{13}$. La Direction Générale de l'Armement (DGA) du Ministère de la Défense, qui étend sa tutelle à la construction aéronautique civile, assure une mise en cohérence du secteur dans la perspective de l'indépendance technologique et militaire du pays et de l'équipement des Armées. À partir des années 1970, l'industrie aéronautique s'est orientée vers le civil, notamment dans le cadre d'alliances internationales, dont Airbus ${ }^{14}$. Les années 1990 et 2000 ont été également marquées par un mouvement d'autonomisation vis-àvis de l'État, notamment par le biais de privatisations, un renforcement des stratégies d'expansion internationale des entreprises et de nombreuses fusions.

Nous montrons que la montée en puissance des directeurs financiers s'observe par l'évolution de leur position dans l'organigramme et les comités exécutifs, tandis que leur professionnalisation ${ }^{15}$ se fait en plusieurs temps. La comparaison des cas de la défense et de l'énergie permet de faire émerger un modèle de transition graduelle des directions financières sur la période $1985-2015^{16}$. Les directions financières des firmes sont tout d'abord occupées essentiellement par des inspecteurs des finances ou, dans certains cas, des ingénieurs sans formation financière (1). Ces pantoufleurs passent directement de l'État à des postes de directeurs financiers qui correspondent à des positions relativement subalternes dans les entreprises. Ensuite s'opère une professionnalisation et une montée en puissance des directeurs financiers (2) qui s'observe par une participation plus systématique aux comités exécutifs. Dans la défense, ces nouveaux directeurs financiers proviennent généralement des écoles de commerce les plus prestigieuses; ils ont effectué leur carrière dans des institutions financières (banques d'affaires, cabinets d'audits, etc.) puis dans les directions financières d'entreprises mais pas immédiatement à des postes de directeurs financiers.

(1924-1985), devenue Total (1985-), ensuite fusionné avec Elf (1976-2000) ; ainsi que les groupes parapétroliers Coflexip (1972-2001) et Technip (1958-).

${ }^{11}$ A. Beltran et J.-P. Williot, Le noir et le bleu: 40 ans d'histoire de Gaz de France, Paris, Belfond, 1998 ; G. Hecht, Le rayonnement de la France: Énergie nucléaire et identité nationale après la seconde guerre mondiale, Paris, 2004, La Découverte; J.-F. Picard, A. Beltran et M. Bungener, Histoires de l'EDF. Comment se sont prises les décisions de 1946 à nos jours?, Paris, Dunod, 1993.

${ }^{12}$ Dans l'aéronautique et la défense, notre panel comprend : Thomson-CSF (1968-2000), devenu Thales (2000-); Snecma (1946-2005), devenu Safran (2005-); et Aerospatiale (1978-1999), devenue EADS (1999-2014), puis Airbus (2014-). Nous excluons de l'analyse Dassault, dont le capital est privé sur toute la période, et les arsenaux, tels que la DCN, GIAT et SNPE.

${ }^{13}$ P. Fridenson et P. Griset, Entreprises de hautes technologies. État et souveraineté depuis 1945, Paris, Comité pour l'histoire économique et financière de la France, 2013 ; M.-D. Seiffert (dir.), «L'industrie aéronautique mondiale, entre ancrage étatique et globalisation », Entreprises et histoire, 73, nº 4, 2013, p. 7-169.

${ }^{14}$ P. Muller, Airbus, l'ambition européenne. Logique d'État, logique de marché, Paris, L'Harmattan, 1989.

${ }^{15} \mathrm{~N}$. Berland et M. Redon, "Qui sont les DAF, ces acteurs de la financiarisation? », Toulouse, 36ème congrès de l'AFC, mai 2015.

${ }^{16}$ La périodisation est indicative, tant elle varie d'une entreprise ou d'un secteur à l'autre. 
Dans l'énergie, cette professionnalisation est moins forte, avec des ingénieurs qui se spécialisent progressivement dans la finance au cours de longues carrières internes.

À la fin de cette phase, les directeurs financiers professionnalisés alternent positions dans des entreprises financières et positions dans la finance d'entreprise ; ils passent ensuite d'une firme à l'autre, à des postes de directeur financier (3). Ils peuvent atteindre des postes de directeur général délégué (c'est-àdire mandataire social au même titre que le PDG). Dans certains cas, cette ultime phase de professionnalisation s'accompagne d'une internationalisation par le recrutement de directeurs financiers étrangers, ou de français passés par des banques d'affaires anglo-saxonnes. Enfin, et c'est sans doute le plus inattendu au vu de la littérature, les années 2010, notamment dans la défense, donnent à voir un retour à des profils plus proches de la première période : pantoufleurs ou ingénieurs qui ne sont pas passés par les investisseurs institutionnels. Ils sont néanmoins plus professionnalisés que dans la première période, notamment via des carrières plus complexes leur permettant de cumuler des capitaux dans différents champs. Nous faisons l'hypothèse que ce retour à des carrières moins spécialisées peut s'interpréter comme la fin d'une phase de financiarisation des firmes.

Les différences entre firmes dépendent du rythme ${ }^{17}$ de leur privatisation dans le cas d'anciennes entreprises publiques et du degré de leur exposition internationale - via les marchés financiers ou leur implantation dans d'autres pays. Ce processus de transformation des carrières et du rôle du directeur financier est lié à la privatisation des entreprises, mais aussi aux transformations des actionnariats, notamment la présence ou non des investisseurs institutionnels anglo-saxons, et à la conception de contrôle des firmes défendues par l'État actionnaire. En effet, la doctrine dominante de gouvernance des entreprises publiques au sein l'État évolue dans le sens d'une financiarisation, en particulier sous l'impulsion de 1'Agence des Participations de l'État (APE) ${ }^{18}$ créée au sein du Ministère des Finances en 2004. Le passage d'un contrôle partagé entre ministères techniques et ministère des finances à une position dominante du ministère des finances au travers de l'Agence des Participations de l'État participe à la modification du profil des directeurs financiers. Étudier la carrière et la position organisationnelle des directeurs financiers est ainsi un moyen de comprendre les transformations générales des stratégies financières des entreprises publiques ou anciennement publiques et des contraintes actionnariales avec lesquelles elles doivent jouer.

\section{DES DIRECTIONS FINANCIERES SUBALTERNES ET PEU SPECIALISEES}

La première période correspond peu ou prou aux années 1980. Les entreprises considérées sont alors très liées à l'État par leur capital mais aussi au travers de la carrière de leurs dirigeants. Les directeurs financiers, qui demeurent à des positions relativement subalternes, ne sont pas issus de

\footnotetext{
${ }^{17}$ Les différentes entreprises n'ont pas été privatisées au même moment, mais également pas de la même manière. Loin d'être un événement ponctuel, la privatisation prend souvent la forme d'un processus long et heurté marqué par des ouvertures progressives de capital et des fusions. La part de l'État baisse ainsi dans le capital, sans que l'État privatise intégralement, ce qui amène à des situations ambiguës où l'État possède $30 \%$ ou $15 \%$ du capital, ce qui assure à l'État un contrôle tout en étant passé sous la barre symbolique des $50 \%$.

${ }^{18} \mathrm{H}$. Coutant et S. Viallet-Thévenin, «The Agence des Participations de l'Etat: towards a financialization of the Shareholding State in France », Lyon, Congrès de Society for the Advancement of Socio-Economics, juillet 2017.
} 
l'industrie financière ou de la finance d'entreprise. Leur accession à cette position est permise par leur passage par les corps financiers de l'État ${ }^{19}$ ou des carrières internes non spécialisées.

\subsection{Ressources d'État ou carrières internes : des directeurs financiers non spécialistes}

Dans les années 1980, le rôle de la direction financière semble relativement mineur dans beaucoup de grandes entreprises des secteurs étudiés. Les directeurs financiers sont souvent directeurs des services financiers et juridiques et ne font pas systématiquement partie des comités de direction. C'est le cas chez Aérospatiale, Total, Electricité de France ou Gaz de France, où les directeurs chargés des finances sont promus au comité exécutif au milieu des années 1980, après quelques années d'exercice. Ainsi, Emmanuel Hau, directeur financier d'EDF depuis 1985 siège seulement à partir de 1990 au comité exécutif. Alain Brion, directeur financier de la Compagnie Française des Pétroles (CFP, ancêtre de Total) attend six ans avant de participer aux comités exécutifs, à partir de 1985. Sur les rapports annuels de la plupart des entreprises (Gaz de France, Thomson-CSF), le directeur financier n'est pas évoqué jusqu'en 2000.

Jusqu'au début des années 1990, à l'exception de Snecma, les directeurs financiers sont d'anciens hauts fonctionnaires, peu spécialisés dans la finance d'entreprise. Emmanuel Hau, directeur financier d'EDF était préfet jusqu'en 1977. Après avoir dirigé un centre de distribution, occupé des postes de responsable des services de l'information puis des affaires publiques, il devient directeur financier. Ce type de parcours n'est pas propre aux EPIC ${ }^{20}$ que sont EDF et Gaz de France. Alain Brion, directeur financier de la CFP de 1979 à 1990, est issu du corps des Mines. Sa carrière à la Direction des Hydrocarbures du Ministère de l'Industrie puis comme conseiller technique au ministère des transports et à la présidence de la République le mène à devenir directeur financier adjoint en 1975 puis directeur financier de la CFP en 1979. Christian Aubin, directeur financier de Thomson-CSF de 1982 à 1987 est un inspecteur des finances qui a pantouflé au bout de 10 ans comme directeur financier, chez Schneider puis chez Thomson. Le même type de profils se retrouve chez Aérospatiale avec des hauts fonctionnaires issus de l'Inspection Générale des Finances (Michel Euvrard, 1974-1983) et de la Cour des Comptes (Jean Picq, 1983-1986). Cela laisse entendre, à une époque où l'entreprise est publique, que la direction financière est peu professionnalisée, puisque le statut d'inspecteur des finances ou de membre de la Cour des Comptes suffit pour y être nommé sans qu'une carrière de quelques années dans la finance d'entreprise ne soit nécessaire.

L'autre type de profil de directeur financier correspond à des ingénieurs issus de carrières internes aux entreprises, qui ne se sont pas spécifiquement spécialisés en finance d'entreprise. Le poste de directeur financier est alors une étape dans une carrière de dirigeant, entre des postes opérationnels et des postes de direction générale, notamment pour des ingénieurs qui ne sont pas issus des grand corps techniques. Jean Sollier, chez Snecma, est diplômé de Télécom Paris, ce qui n'est pas la formation dominante dans cette entreprise dirigée par des polytechniciens, dont beaucoup issus du corps de l'armement. Il a fait toute sa carrière chez Snecma dans des postes managériaux peu techniques mais prestigieux, telle que la direction du programme Concorde ou de la joint-venture CFM International avec General Electric. Le poste de directeur financier s'inscrit dans cette trajectoire de direction qui le mène

\footnotetext{
${ }^{19}$ Cette pratique de recrutement remonte dans les grandes entreprises industrielles au début du XXème siècle pour l'inspection des finances. Voir : H. Joly, Diriger une grande entreprise au XXe siècle : L'élite industrielle française, Tours Université François Rabelais, 2013.

${ }^{20} \mathrm{EPIC}$ : établissement public à caractère industriel et commercial.
} 
ensuite au poste de PDG d'une des filiales du groupe Snecma. Dans une entreprise d'ingénieurs techniques comme Snecma, sa carrière est typique des ingénieurs considérés comme les moins techniques $^{21}$, avec des dimensions gestionnaires et organisationnelles importantes. Gestion et finance apparaissent regroupées dans un même ensemble cohérent dans les carrières. Ce type de carrières se retrouve encore dans les années 1990 dans l'énergie avec Bruno Weymuller, directeur financier d'Elf de 1994 à 2000, qui alterne fonctions opérationnelles et fonctions financières au sein du groupe.

Les contacts antérieurs des directeurs financiers des années 1980 avec le monde de la finance sont souvent des plus ténus. Ces carrières reflètent à la fois le fait que la direction financière est peu professionnalisée et que l'État est l'actionnaire prépondérant. La finance d'entreprise n'est vue au plus que comme l'une des compétences qu'il est bon qu'un dirigeant maîtrise.

\subsection{La direction financière comme interface avec l'État}

Au début des années 1980, les entreprises considérées sont largement à capitaux publics, à l'exception de Total, dont $60 \%$ du capital est privé. Cependant, même pour Total et Elf, le rapport à l'État reste très marqué :

«De 1976 à 1994, il y a une situation intermédiaire, avec des préoccupations vis-à-vis des marchés, on côte Elf à New York, et de l'autre côté, des relations très fortes avec la tutelle publique [...] Moi, je suis arrivé en 1981, la privatisation [n'était pas encore faite]. On avait un système encore assez consanguin. Avec une forte délégation opérationnelle, industrielle à la société. Le tuteur s'exprimait dans le conseil de l'ERAP22, ou étaient représentés le Trésor, le Budget, l'industrie et les Affaires Étrangères. C'était un instrument de pilotage ou se décidait la stratégie » (Entretien avec un ancien directeur financier d'Elf, juillet 2013).

Si l'État a financé les politiques d'investissements lourds menées par les entreprises de l'énergie, il a cessé dans les années 1970. EDF et Gaz de France demeurent des EPIC jusqu'en 2004, mais l'État introduit durant cette période le principe d'une rémunération du capital, c'est-à-dire l'apparition de flux financiers depuis l'entreprise vers l'État. À partir du début des années 1980, l'administration, en concertation avec les directions d'EDF et Gaz de France, a fixé des taux de rémunération de l'État dans les contrats liant l'État aux établissements publics pour des périodes de trois ans.

"Progressivement, le FDES ${ }^{23}$ n'a plus rien drainé vers nous. Ensuite, à partir de 1980, les dotations en capital elles-mêmes ont disparu. Et nous nous retrouvons avec un simple système de tutelle et non plus un système de financement faisant participer l'État-propriétaire ${ }^{24}$ (Hau, directeur financier d'EDF).

Thomson, entreprise nationalisée en 1982, et Snecma, nationalisée en 1946, sont toutes deux restées pendant toute cette période des Sociétés Anonymes, avec l'État pour actionnaire. Il est notable que lorsque les entreprises ont un résultat net négatif (l'essentiel des années 1980 pour Thomson comme pour

${ }^{21}$ H. Coutant, Un capitalisme d'ingénieurs. Construire un groupe aéronautique après une fusion, Paris, Sciences Po, Thèse en Sociologie, 2016

${ }^{22}$ ERAP : Établissement de Recherche et d'Activité Pétrolière, EPIC qui gérait les participations de l'État dans Elf.

${ }^{23}$ FDES : Fonds de Développement Économique et Social.

24 E. Hau, «Le financement de l'industrie électrique après la Seconde Guerre mondiale », colloque «Le Financement de l'industrie électrique 1880-1980 », Paris, 1994. 
Snecma ${ }^{25}$ ), les entreprises ne versent pas de dividendes, au contraire de ce qui pourra se passer dans les années 2000.

Dans les directions financières des entreprises publiques, le rapport aux marchés financiers et aux dividendes évolue dans les années 1980 mais, pour autant, l'État reste central dans les rapports actionnariaux. Il contrôle par ailleurs une grande partie de l'économie, notamment au travers de son large secteur public. Dans ce contexte, les directeurs financiers sont très majoritairement issus de la haute fonction publique, comme un grand nombre de dirigeants exécutifs ${ }^{26}$. La direction financière prend de l'importance durant cette période, sans pour autant que ses dirigeants ne fassent partie, comme aux ÉtatsUnis à la même époque, de l'équipe des deux ou trois plus proches collaborateurs du PDG.

\section{LA PROFESSIONNALISATION DE LA DIRECTION FINANCIERE}

Au cours des années 1990 et au début des années 2000, suivant des temporalités différentes selon les secteurs et les entreprises, les carrières des directeurs financiers évoluent, et suivent un processus de professionnalisation. Les parcours des dirigeants financiers se différencient d'autres parcours de dirigeants, et des carrières spécifiques aux directeurs financiers apparaissent dans le cas de l'industrie de défense. L'évolution des carrières participe des transformations des entreprises durant cette période.

\subsection{Spécialisation et renforcement de la fonction financière}

Le rôle du directeur financier s'est considérablement accru dans les années 1990 au sein des grands groupes industriels publics, particulièrement dans l'énergie. Emmanuel Hau, Directeur des services financiers et juridiques d'EDF en 1985, devient directeur général adjoint (1994), puis Directeur général délégué en 1995. Gérald Arbola, Directeur financier de la Cogéma, entre au directoire en 2001, à la création d'Areva, puis devient Directeur général délégué en 2006. Il figure ainsi parmi les quatre plus importants dirigeants de l'entreprise nucléaire. Chez Gaz de France, le directeur financier apparaît au sein de la direction (sans pour autant faire partie du comité exécutif), et se rapproche du premier cercle constitué autour du PDG ${ }^{27}$.

Cette montée en puissance s'accompagne d'une professionnalisation de la fonction de directeur financier, selon deux processus différents selon les entreprises concernées. Le premier processus, visible dans l'énergie, consiste en une autonomisation des directions financières vis-à-vis d'autres directions de l'entreprise et de l'État, dans le cadre de carrières internes. Plus rarement liés à l'État, les directeurs

${ }^{25}$ Il est difficile de reconstituer des séries complètes de versement de dividendes, notamment pour Thomson dont le périmètre a changé à plusieurs reprises au cours des années 1980 et 1990.

${ }^{26} \mathrm{~S}$. Viallet-Thévenin, «État et secteur énergétique en France : quels dirigeants pour quelles relations ? , Revue française de sociologie, 56, n³, 2015, p. 469-499.

27 Ce cas se rapproche des Arsenaux, en particulier la Direction des Chantiers Navals qui était encore une administration. Ses cadres dirigeants sont des ingénieurs de l'armement, et il n'existe pas de fonction de directeur financier, ou de direction financière. Aucune différence n'existe entre le budget général de l'État et celui de la DCN. Sa transformation en entreprise publique en 2003 sous le nom de DCNS amène à la création d'un poste de directeur financier pour lequel est recruté Jean des Courtis, un directeur financier extérieur à l'entreprise, diplômé de l'ESCP, qui a fait une carrière dans la finance d'entreprise. DCNS offre le cas d'une entreprise passée directement d'une administration à une professionnalisation relativement avancée des directeurs financiers, sans phase intermédiaire. 
financiers des années 1990 ont fait la majeure partie de leur carrière dans l'entreprise dont ils deviennent directeurs financiers. Une première frontière se créée avec la haute fonction publique : ces derniers se différencient des hauts fonctionnaires des finances ou des ingénieurs des grands corps d'État responsables de la tutelle technique des entreprises publiques. Robert Castaigne, directeur financier de Total de 1994 à 2008 est diplômé de l'ENSPM (École Nationale Supérieure des Pétroles et Moteurs) et de l'École Centrale de Lille et titulaire d'un doctorat en économie. L'essentiel de sa carrière se déroule dans les services économiques puis financiers de Total. Dans l'énergie, le directeur financier, issu d'une école de commerce, a fait une longue carrière au sein des directions financières de l'entreprise. Ainsi apparaissent, à l'échelle des entreprises, des différences entre les parcours des directeurs financiers et ceux des autres membres de la direction, constituant autant des frontières locales ${ }^{28}$. Internes à l'entreprise, partageant certaines caractéristiques des autres membres de la direction - longue carrière dans l'entreprise, postes opérationnels, diplôme d'ingénieur - les directeurs financiers se sont progressivement spécialisés dans la finance d'entreprise.

Le second processus est plus radical, avec une autonomisation des directeurs financiers y compris vis-à-vis de l'entreprise. Il correspond, dans les entreprises de la défense, au recrutement de directeurs financiers issus d'écoles de commerce, ayant débuté leurs carrières dans la finance ou dans l'audit, avant de faire carrière dans les directions financières d'entreprises de tailles croissantes. C'est le cas d'Alain Hagelauer, directeur financier de Thomson de 1987 à 2000. Issu d'une école de commerce (HEC), il a débuté sa carrière dans l'audit (dix ans chez Arthur Andersen), avant de faire carrière dans la finance d'entreprise dans des entreprises de taille croissante (Continental Grain puis Gervais-Danone) et avec plusieurs postes successifs (contrôle de gestion puis finance). Chez Thomson même, il fait carrière pendant cinq ans dans la direction financière avant d'en prendre la direction. On retrouve des parcours similaires à la Snecma avec Alain Amiot, diplômé d'HEC, passé par les banques CCF et Worms avant de devenir Directeur Financier de Snecma, et Noël Gauthier, diplômé de l'ESCP, qui a débuté sa carrière comme analyste financier avant de passer dans la finance d'entreprise, chez Renault puis Snecma. Dans l'industrie de défense, la rupture avec le modèle antérieur est plus nette que dans le secteur de l'énergie. La seule exception est l'Aérospatiale, probablement due à ses liens encore plus forts à l'État. Les directeurs financiers demeurent jusqu'à la fin des années 1990 d'anciens hauts fonctionnaires. Le seul élément de professionnalisation, entre l'administration et le poste de directeur financier, dans le parcours de François Thomazeau (1987-1991) et François Auque (1991-2000), est un bref passage de quelques années dans des entreprises financières (Crédit Agricole ou Suez). Le renforcement de la holding EADS dans les années 2000 puis Airbus dans les années 2010 amène la nomination de directeurs financiers allemands, dont la carrière est essentiellement interne à la branche allemande, DASA, mais spécialisée dans la finance d'entreprise.

Les carrières des directeurs financiers ont une tendance à la complexification et à l'accumulation de ressources plus diverses. Ces ressources peuvent être accumulées au cours de carrières internes, en alternant postes opérationnels et financiers - comme chez Total, Elf ou Technip - ou déjà spécialisées en finance d'entreprise - comme à la Cogéma ou chez EDF. Dans la défense, cette spécialisation est plus forte, grâce au passage par différentes entreprises dont des firmes financières. La direction financière se

\footnotetext{
${ }^{28}$ A. Abbott, « Things of Boundaries », Social Research, 62, nº4, 1995, p. 857-882.
} 
singularise des autres directions - notamment opérationnelles - qui recrutent encore largement d'anciens hauts fonctionnaires dans les années 1990-2000.

\subsection{La direction financière comme interface avec les marchés financiers et support des transformations des firmes}

Dans les années 1990, les entreprises publiques de l'énergie et de la défense sont confrontées à de grandes transformations de leur environnement institutionnel. Les liens capitalistiques avec l'État sont progressivement dissous pour les pétroliers (Total et Elf), privatisés de 1992 à 1994, ainsi que pour les parapétroliers (Technip et Coflexip). Dans le cas d'EDF, de Gaz de France, ainsi que de la Cogéma, l'enjeu devient l'affichage d'une distance avec l'État, alors que leur statut d'entreprises publiques contrarie leur ambition d'internationalisation, ce qui a pour effet de faire diminuer le nombre d'anciens hauts fonctionnaires au sein des instances gouvernantes ${ }^{29}$.

Du fait de la transformation de la structure de leur actionnariat, les pétroliers et parapétroliers entretiennent des relations progressivement déterminantes et exclusives avec des investisseurs institutionnels anglo-saxons. Le directeur financier est au centre de ces transformations à travers une communication financière accrue, une politique de dividende fort et une place plus centrale dévolue aux outils financiers dans la gouvernance de l'entreprise.

"La grande date, c'est la privatisation de 1994, beaucoup plus forte que Total pour lequel la privatisation a été progressive. Nous, on est passés de 60 à 0 . C'était une très grosse opération, très bien menée, je peux le dire, je suis arrivé après à la direction financière. Là, on a eu tous les problèmes financiers, juridiques du droit anglo-saxon. Ensuite, on a mis en place une communication financière, avec des présentations spécialisées pour les actionnaires, ce qu'on appelait des road shows. On était très en avance, les pétroliers, de vingt ans sur des entreprises françaises comme Orange, Renault, qui le font aussi maintenant.» (Entretien avec un ancien directeur financier d'Elf, juillet 2013)

En revanche, dans les autres entreprises de l'énergie ou à l'Aérospatiale, l'actionnaire et interlocuteur principal des directions financières reste l'État. EDF et Gaz de France rémunèrent l'État via le versement de dividendes à partir du début des années 1980, mais, jusqu'en 2005, date de leur privatisation partielle, ces versements demeurent très modestes. A contrario, les entreprises de défense gardent un capital essentiellement public mais la privatisation est une perspective à moyen terme. La stratégie de la direction consiste donc à professionnaliser la gestion des finances de l'entreprise en vue d'une ouverture du capital.

Durant les années 1990 et le début des années 2000, les entreprises des différents sous-secteurs de l'énergie et de la défense ont en commun un changement de leur morphologie, par leur regroupement ou le rachat d'autres entreprises. Pour les entreprises de l'énergie, fragilisées par les mouvements de libéralisation des marchés de l'électricité et du gaz, ainsi que par leur privatisation, le but est de préserver leurs positions au sein de leurs marchés. Thomson-CSF, Aérospatiale et Snecma connaissent eux aussi de profondes transformations de gouvernance, de périmètres, et s'internationalisent dans un contexte de consolidation des entreprises de défense. Les directeurs financiers de Snecma et Thomson-CSF sont restés à leur poste longtemps, entre douze et quatorze ans, assurant une stabilité de la direction financière pendant ces transformations. Dans les opérations de fusions-acquisitions, la direction financière et son

\footnotetext{
${ }^{29}$ S. Viallet-Thévenin, «État et secteur énergétique en France : quels dirigeants pour quelles relations ? », op. cit.
} 
expertise jouent un rôle prépondérant, ce qui donne de l'importance au directeur financier. La direction financière de ces grands groupes apparaît comme un poste qui ne prépare pas à d'autres postes de direction générale mais assure une crédibilité financière à des entreprises en profonde transformation financière, stratégique et actionnariale. La variation entre firmes dépend du rythme de leur privatisation dans le cas d'anciennes entreprises publiques et du degré de leur exposition aux marchés financiers internationaux.

Au terme de ce processus, les carrières des directeurs financiers dessinent la juridiction de la direction financière, et, plus particulièrement, celle des directeurs financiers. Celle-ci s'étend du contrôle de gestion aux fusions acquisitions, et apparaît ainsi bien plus large et centrale que celle des directions financières des années 1970-1980, qui étaient centrées sur la comptabilité, et répondaient à des logiques budgétaires plus proches de celles de l'État. Les transformations qui affectent les entreprises publiques dans les années 1990 renforcent la direction financière qui devient l'épicentre de la direction tandis que le directeur financier devient l'un des plus proches conseillers du PDG.

\section{AUTONOMISATION DES CARRIERES DES DIRECTEURS FINANCIERS}

La professionnalisation des directeurs financiers connaît dans les années 2000 une seconde phase marquée par une autonomie accrue vis-à-vis des entreprises à travers des parcours de Chief Financial Officers parfois internationaux qui circulent d'une entreprise à l'autre sans carrières interne. La recherche de légitimité financière est alors essentielle pour des entreprises dont l'exposition et la dépendance aux investisseurs institutionnels et aux marchés financiers s'accroît. Cependant, cette phase se clôt, dans la défense, par un retour à des individus passés par l'État ou aux longues carrières internes à de grands groupes industriels, ce qui invite à s'interroger sur une possible fin de cycle de financiarisation de ces entreprises.

\subsection{Des Chief Financial Officers professionnels et internationaux}

Les directeurs financiers des années 2000 sont de plus en plus des individus spécialisés dans les fonctions de directeur financier. Ils ont fait l'essentiel de leur carrière dans le monde de la finance : dans l'audit, chez des investisseurs institutionnels puis souvent dans de grandes entreprises à des fonctions de direction financière. Cette phase ultime de professionnalisation est marquée par deux phénomènes: l'apparition de carrières par lesquelles des individus occupent des fonctions de directeur financier dans plusieurs entreprises successivement, et leur internationalisation. Un parcours au sein de l'entreprise ne constitue plus un préalable nécessaire à l'accession au poste de directeur financier. Une fois atteint un poste de directeur financier, ils passent d'une entreprise à l'autre, généralement de taille croissante. De nouveaux passages en banque d'affaire sont possibles entre deux postes de directeurs financiers. Les directeurs financiers nommés dans les années 2000 proviennent tous de l'extérieur de l'entreprise au sein de laquelle ils occupent un poste. Olivier Dubois, diplômé de l'ESSEC, de l'IEP de Paris et licencié ès sciences économiques, commence sa carrière à la Banque Paribas, où il devient fondé de pouvoir au bout de dix ans. Il rejoint ensuite en 1991 la direction financière de filiales du groupe Spie où il parvient au poste d'administrateur directeur général adjoint. Il devient directeur général finances et contrôle du groupe 
chez Technip en $2002^{30}$. Cette transition est opérée en 2003 chez EDF, en 2007 chez Areva et en 2008 chez Gaz de France, à l'occasion de sa fusion avec Suez. Le même processus s'observe dans l'aéronautique et défense, en 2000 chez Thales et en 2009 chez Safran. L'internationalisation des profils vient s'ajouter à cette professionnalisation, parfois dans un second temps. Ainsi, les directions financières d'Engie à partir de 2015 et de Technip à partir de 2008 sont occupées par des étrangers : l'étatsunien Julian Waldron pour Technip, et l'autrichienne Judith Hartmann pour Engie. Dans ces entreprises majoritairement masculines, la direction financière est une des premières à se féminiser : l'américaine Maryann Mannen succède en 2015 à Julian Waldron ; Judith Hartmann succède à Isabelle Kocher, qui était directrice financière d'Engie depuis 2011.

Thales et Safran ont la particularité d'avoir accompli ce tournant d'internationalisation et professionnalisation à neuf ans d'intervalle mais à travers le même individu, Ross McInnes, nommé directeur financier de Thales en 2000 puis de Safran en 2009. McInnes est une incarnation de ces transformations : australien d'origine, diplômé d'Oxford, il fait la première partie de sa carrière en banques d'affaires internationales à Londres, Rio de Janeiro, Paris et Chicago. Ensuite il devient directement directeur financier dans l'industrie agro-alimentaire puis chez Thales. S'il partage avec son prédécesseur Alain Hagelauer plusieurs caractéristiques indiquant sa professionnalisation (banques d'affaires puis finance d'entreprises) McInnes se différencie par une plus grande internationalisation et un passage par les banques d'affaires anglo-saxonnes. Il n'a par ailleurs pas eu besoin de faire ses preuves en interne, puisqu'il est directement recruté comme directeur financier. Après cinq ans chez Thales, il est brièvement nommé directeur financier de PPR et refait un passage dans la finance comme vice-chairman de la branche européenne du gestionnaire de fonds australien Macquarie Capital. Il est recruté chez Safran en remplacement de Noël Gauthier qui était à ce poste depuis quinze ans, dans une période où le capital du groupe s'internationalise. Sa position est supérieure à son prédécesseur puisqu'il est immédiatement membre du directoire avant de devenir Directeur Général Délégué. Le cas de McInnes est à la fois emblématique et extrême, dans le sens où il concentre plusieurs transformations : passage par les banques d'affaires, passage d'une entreprise à l'autre et internationalisation tournée vers le monde anglo-saxon. Il est aussi emblématique du fait qu'un poste de directeur financier peut être un moyen d'accéder au sommet des entreprises, une nouveauté qui montre leur nouvelle centralité. Lors du départ du PDG, Jean-Paul Herteman, en 2015, le poste de PDG est scindé entre un poste de directeur général et un poste président du Conseil d'Administration, qui revient à McInnes. De même, dans l'énergie, Isabelle Kocher, nommée directrice financière de GDF-Suez en 2011, a été nommée en 2015 directrice générale des opérations, puis directrice générale en 2017. Jusqu'alors, aucun directeur financier n'était devenu PDG dans le secteur de l'énergie français. Par ailleurs, dans l'énergie, le directeur financier n'est pas le seul au sein du comité exécutif à représenter le champ de la finance et les intérêts du nouvel actionnariat. Sa position est renforcée par le directeur de l'audit et des risques, souvent aussi issu du même type de carrière.

\subsection{La recherche d'une légitimité financière}

Cette dernière phase de professionnalisation peut s'expliquer par une modification de l'actionnariat des firmes, une diffusion de la valeur actionnariale et un besoin pour les entreprises d'assurer leur légitimité financière vis-à-vis des investisseurs institutionnels. La sociologie néo-

\footnotetext{
30 Après son départ de Technip en 2007, il prendra successivement la direction financière de Theolia, du groupe CMA-CGM, d'Alvarez \& Marsal puis du groupe Elior.
} 
institutionnaliste américaine a montré que les entreprises qui ne répondent pas aux standards financiers sont sous-évaluées ${ }^{31}$, attaquées ${ }^{32}$ et sont l'objet de pressions par les investisseurs et analystes ${ }^{33}$. Avec la montée des actionnaires institutionnels au sein de l'actionnariat, prendre un directeur financier issu de l'entreprise ne suffit souvent plus pour les satisfaire, et les directions recourent dans la majorité de situations à un dirigeant externe, dont le sérieux a été validé sur le marché international du travail des directeurs financiers, et qui a une forte connaissance des investisseurs institutionnels pour y avoir travaillé. Dans leur pratique professionnelle, ces directeurs financiers travaillent à assurer la crédibilité de leur entreprise, notamment par des road shows ${ }^{34}$ à destination des investisseurs, par une communication financière clarifiée et renforcée, par une mise aux normes comptables IFRS (International Financial Reporting Standards) ou encore une meilleure couverture aux risques de change. Par ailleurs, la tutelle exercée par l'État évolue dans le même sens, par l'intermédiaire de l'Agence des Participations de l'État, créée en 2004, dont les pratiques et l'idéologie sont proches de celles d'investisseurs institutionnels. Comme le dit un des administrateurs de Safran en parlant de la «confiance» du marché, le directeur financier construit la légitimité financière du groupe :

«Et puis il y a eu l'embauche de Ross McInnes, qui a été fondamentale.

$Q:$ Pourquoi ?

Il a assuré la couverture au dollar. [...] Et puis il a eu une politique vis-à-vis des actionnaires qui a fait monter le cours.

$Q:$ Comment ça?

La confiance! [...] La confiance est fondamentale. [...] Le marché, avec Ross, a repris confiance. » (Administrateur de Safran, décembre 2013)

Dans la défense, cela s'accompagne de politiques de soutien du cours de l'action et de versement de dividendes pas nécessairement supérieurs mais plus stables en proportion du résultat net. La croissance du cours de l'action de Safran, de 400\% entre 2009 et 2013, est un indicateur de l'efficacité de cette stratégie de légitimation de la finance de l'entreprise vis-à-vis des investisseurs.

Si on met de côté les opérateurs pétroliers et parapétroliers qui ont été exposés aux pratiques des investisseurs institutionnels dès les années 1990, le capital des entreprises de l'énergie (EDF, Gaz de France, Areva) est destiné à être privatisé - du moins en partie - dans les années 2000. Ainsi, au sein des équipes dirigeantes comme du ministère de l'économie, divers projets d'évolution de l'actionnariat se succèdent, jusqu'à l'adoption de la loi de 2004 portant transformation des statuts d'EDF et de Gaz de France et l'ouverture de leur capital en 2004 et 2005. En revanche, en dépit de nombreux projets de la direction, le capital d'Areva n'est ouvert en 2009 qu'à Mitsubishi Heavy Industries, un partenaire

${ }^{31}$ E. Zuckerman, «The Categorical Imperative: Securities Analysts and the Illegitimacy Discount », American Journal of Sociology, 104, n5, 1999, p. 1398-1397.

${ }^{32}$ G. Davis et S. Stout, « Organization Theory and the Market for Corporate Control: A Dynamic Analysis of the Characteristics of Large Takeover Targets, 1980-1990», Administrative Science Quarterly, 37, nº4, 1992, p. 605-633.

${ }^{33}$ M. Useem, Investor Capitalism, New York, Basic Books, 1996.

${ }^{34}$ Ibid. 
industriel japonais. Ces projets ont été préparés largement en amont, et le recrutement de directeurs financiers extérieurs à l'entreprise et professionnalisés dans cette fonction peut se comprendre comme une manière d'apparaître comme des entreprises ouvertes aux pratiques des investisseurs institutionnels et attentives aux intérêts des actionnaires minoritaires, constitués principalement par ces mêmes acteurs financiers. Au cours de cette période, le montant des dividendes augmente massivement, et passe par exemple pour EDF de 300 millions d'euros par an en 2003 à trois milliards d'euros en 2006.

\subsection{Vers une domestication de la finance?}

Notre récit aurait pu s'achever là. Cependant, la période la plus contemporaine, les années 2010, est marquée, en particulier dans l'aéronautique et la défense, par une évolution paradoxale. Après le relatif paroxysme incarné par Ross McInnes, ont été nommés, tant chez Thales dans les années 2000 que chez Safran dans les années 2010, des directeurs financiers d'un genre différent, plus similaires aux périodes antérieures. Tout d'abord, on assiste à un retour du capital d'État avec un préfet, Patrice Durand (20052012), un énarque qui n'a pas la légitimité financière d'un Inspecteur de Finances, mais qui a rapidement bifurqué dans sa carrière administrative de quinze ans vers les finances publiques et notamment les administrations financières en lien avec les entreprises publiques (Trésor et Service des Participations de l'État). La suite de sa carrière est une accumulation éclair des capitaux nécessaires pour devenir directeur financier : finance d'entreprise (chez Air France durant trois ans) puis banques (au Crédit Lyonnais et au Crédit Agricole, trois ans en tout) avant d'être recruté directement comme Directeur Financier de Thales. Chez Safran, après McInnes, est nommé en 2015 Bernard Delpit, un inspecteur des finances. Il multiplie les ressources par une circulation entre l'État (à Bercy et à l'Élysée), la finance d'entreprise (chez PSA et à La Poste), et la banque (au Crédit Agricole), avec des allers-retours entre État et entreprises et une montée dans la hiérarchie financière. Ces deux individus cumulent donc les ressources des directeurs financiers professionnels (allers-retours entre finance d'entreprise et finance, absence de carrière interne aux entreprises), mais en les combinant avec des ressources liées à l'État qui avaient précédemment perdu de leur importance : ENA, allers-retours entre administration et entreprises. On peut faire l'hypothèse que ces profils sont une réponse à la financiarisation et professionnalisation de l'APE, puisque Durand est passé par son ancêtre, le Service des Participations de l'État et par les finances d'une entreprise publique, Air France, de même que Delpit, qui a été Conseiller économique à l'Élysée et directeur financier d'une entreprise publique, la Poste.

Enfin, le dernier directeur financier de Thales en date, depuis 2012, incite à aller plus loin en formulant l'hypothèse d'une fin de cycle de financiarisation de l'entreprise. Pascal Bouchiat est d'abord un ingénieur issu d'une école modeste (l'ESCIL de Lyon) mais complété d'un MBA de l'ESC Lyon puis d'un Executive MBA LSE/HEC/NYU, mais surtout il a fait la totalité de carrière dans un groupe industriel, Rhône Poulenc puis Rhodia. Il débute comme ingénieur de R\&D et de production avant de passer à la finance d'entreprise, sans doute par le biais de carrières internes destinées à sélectionner de futurs dirigeants. Il finit directeur financier de Rhodia, tâche dont il semble s'acquitter avec succès puisqu'il est cité dans le presse financière comme l'un des meilleurs directeurs financiers en poste en $2008^{35}$. Il est ensuite recruté directement au poste de directeur financier de Thales. On constate donc le retour d'un profil de financier d'entreprise ayant peu changé d'entreprise, n'étant passé ni par l'État ni par la finance,

\footnotetext{
${ }^{35}$ Voir l'Express : https://www.lexpress.fr/emploi/la-finance-a-1-honneur_718515.html
} 
à la formation initiale d'ingénieur. Son cas amène à se demander s'il ne marque pas la fin d'un cycle, et la domestication de la finance par la direction après une longue professionnalisation ${ }^{36}$.

Maintenant plus autonomes de l'État et crédibles financièrement vis-à-vis des investisseurs institutionnels, les directions de ces entreprises totalement ou partiellement privatisées n'ont plus besoin de renforcer leurs ressources et leur image financière, voire sont capables de mieux domestiquer les enjeux financiers, y compris pour les soumettre à des ambitions industrielles et technologiques.

\section{CONCLUSION}

L'histoire des directions financières des entreprises de l'énergie et de la défense donne à voir un renforcement et une professionnalisation croissante des directeurs financiers. Spécialisation dans la finance, puis dans le poste de directeur financier, internationalisation et carrières combinant industrie financière et finance d'entreprise ont profondément transformé le profil des directeurs financiers. Cette transformation des individus et de leur position dans l'organisation a permis aux entreprises partiellement ou totalement privatisées de construire leur légitimité financière. Les entreprises ont pu ainsi s'assurer la confiance des investisseurs institutionnels prenant une part croissante dans leur capital. Elles ont également pu répondre à la financiarisation croissante de l'État actionnaire lui-même sous l'impulsion de l’Agence des Participation de l'État créée par le Ministère des Finances en 2004.

L'histoire des directeurs financiers des groupes à capitaux publics au cours des quarante dernières années éclaire plusieurs transformations du capitalisme français, que celles-ci concernent les élites économiques, la réponse des firmes industrielles à la financiarisation ou la réaction et participation de l'État à la financiarisation de l'économie. Tout d'abord, cette transformation confirme la thèse d'un renforcement et d'une professionnalisation de la fonction financière comme une dimension de la financiarisation. Nous sommes par contre amenés à nuancer la thèse des convertis de la finance développée par Pierre François et Claire Lemercier ${ }^{37}$. Il apparaît que les entreprises ont été chercher de nouvelles ressources pour répondre à la financiarisation, que ce soit dans les business school, les cabinets d'audit ou dans les banques d'affaires internationales. Cependant, la dernière phase que nous avons identifiée, marquée par un retour au capital d'État ou industriel, permet de formuler l'hypothèse que cette recherche de nouveaux capitaux s'affaiblit lorsque que les entreprises ont assuré leur crédibilité financière et sont moins menacées par les marchés financiers. La conversion s'accompagnerait donc d'une forme de domestication de la finance par les élites économiques françaises. Cette conclusion en induit une autre : les tensions entre capital industriel, étatique et financier semblent s'apaiser dans la phase la plus récente. Si les industriels semblent avoir domestiqué la finance, il apparaît aussi que les élites d'État se sont largement converties à la finance. Plus largement, cette conclusion va dans le sens d'une financiarisation

\footnotetext{
${ }^{36}$ Cette hypothèse peut être corroborée, avec certaines limites, par le fait que dans l'énergie, l'entreprise la plus anciennement confrontée aux marchés financiers, Total, est également celle où le directeur financier a fait une carrière interne dans son entreprise. Le parcours de Patrick de la Chevardière, occupant ces fonctions depuis 2008, est typique du profil de directeur financier des années 1990 : diplômé de Centrale et d'HEC, il alterne des fonctions dans le forage, à la direction financière et dans le raffinage. Il faut cependant mettre en regard sa carrière avec les pratiques de la direction de Total, qui ne recrute dans son comité exécutif que des dirigeants internes.

${ }^{37}$ P. François et C. Lemercier, «Une financiarisation à la française (1979-2009), Mutations des grandes entreprises et conversion des élites », op. cit.
} 
de l'État, en particulier au travers des grands corps administratifs et de l'émergence de l'Agence des Participations de l'État. 